\title{
Variation in the choice of elective surgical procedure for abdominal aortic aneurysm in Spain
}

This article was published in the following Dove Medical Press journal: Vascular Health and Risk Management

\author{
M Jesús Quintana ${ }^{1,2}$ \\ Ignasi Gich ${ }^{1-3}$ \\ Julián Librero ${ }^{4,5}$ \\ Sergi Bellmunt-Montoya ${ }^{6,7}$ \\ José R Escudero $3,8,9$ \\ Xavier Bonfill ${ }^{1-3,10}$ \\ On behalf of the AAA \\ Spanish Study Group \\ 'Department of Clinical Epidemiology \\ and Public Health, University \\ Hospital de la Santa Creu i Sant \\ Pau (IIB Sant Pau), Barcelona, Spain; \\ ${ }^{2} \mathrm{CIBER}$ of Epidemiology and Public \\ Health (CIBERESP), Barcelona, Spain; \\ ${ }^{3}$ Universitat Autònoma de Barcelona, \\ Barcelona, Spain; ${ }^{4}$ Navarrabiomed- \\ UPNA -Departamento de Salud, \\ IDISNA, Pamplona, Spain; ${ }^{5}$ Red de \\ Investigación en Servicios de Salud en \\ Enfermedades Crónicas (REDISSEC), \\ Bilbao, Spain; ${ }^{6}$ Department of Angiology, \\ Vascular and Endovascular Surgery, \\ Vall d'Hebron University Hospital, \\ Barcelona, Spain; ${ }^{7}$ Vall d'Hebron \\ Research Institute (VHIR), Barcelona, \\ Spain; ${ }^{8}$ Joint Service of Angiology, \\ Vascular and Endovascular Surgery, \\ Sant Pau-Dos de Maig Hospital, \\ Barcelona, Spain; ${ }^{9} \mathrm{CIBER}$ Cardiovascular \\ Diseases (CIBERCV), Barcelona, Spain; \\ ${ }^{1}$ Iberoamerican Cochrane Centre, \\ Barcelona, Spain
}

Correspondence: Xavier Bonfil Department of Clinical Epidemiology and Public Health, University Hospital de la Santa Creu i Sant Pau (IIB Sant Pau), Iberoamerican Cochrane Centre, C/ Sant Antoni Maria Claret, 167, Pavelló I8, planta 0, Barcelona CP: 08025, Spain

Tel +34935537808

Fax +34935537809

Email xbonfill@santpau.cat
Objective: The two main surgical treatments for abdominal aortic aneurysm (AAA) are open surgical repair (OSR) and endovascular aneurysm repair (EVAR). The aim of this study was to analyze variation among Spanish hospitals in the use of OSR or EVAR for AAA. A secondary aim was to assess changes in preferences for these two procedures over time.

Methods: This was a retrospective longitudinal study based on discharge data from public hospitals in Spain during 2002-2012. Patient inclusion criteria were: age $>18$ years, elective admission, primary diagnosis of unruptured AAA, and surgical treatment with OSR or EVAR. The characteristics of the treating center, patients, and in-hospital mortality were recorded.

Results: We included 16,737 patients from 114 hospitals; 6,809 (40.7\%) underwent EVAR and 9,928 (59.3\%) underwent OSR. The total volume of surgeries increased throughout the period, and the probability that any given procedure was EVAR increased by $20 \%$ per year (OR 1.20 , $P<0.001)$. The volume and distribution of the two procedures varied highly across the participating hospitals. Overall, in-hospital mortality rate was 3.6\% and it decreased during the study period (5.3\% in 2002 and 3.2\% in 2012), mainly due to a decrease in OSR-related mortality, despite a slight increase in EVAR-related mortality. Hospitals with higher surgical volumes were more likely to use EVAR and have lower in-hospital mortality rates.

Conclusion: This study reveals high variability in the surgical treatment of unruptured AAA across Spanish hospitals. The number of interventions has increased in recent years, with EVAR accounting for a growing percentage of these surgical procedures. Overall in-hospital mortality rates decreased significantly during this period, mainly due to lower mortality among patients undergoing OSR. In-hospital mortality rates were lower in higher-volume centers, regardless of the surgical approach used. Further research on variability and appropriateness of surgical management of AAA is required to assess the suitability of concentrating elective AAA repair in more experienced centers to potentially achieve better outcomes.

Keywords: abdominal aortic aneurysm, open surgical repair, endovascular aneurysm repair, variability

\section{Introduction}

Abdominal aortic aneurysm (AAA) is a very severe pathology that presents a high risk of mortality in case of rupture. ${ }^{1}$ Open surgical repair (OSR) has long been the primary treatment option to repair an intact AAA; however, endovascular repair (EVAR) - a minimally invasive procedure - has largely replaced OSR in many hospitals. ${ }^{2-6}$ Indeed, due to recent technological advances, many authors and centers now consider EVAR the procedure of choice for surgical repair of AAA. ${ }^{7,8}$ In recent years, several studies have been conducted to assess trends in the surgical treatment of AAA. Those studies have found a persistent increase in surgical procedure rates 
for AAA - likely due to population aging, among other factors. ${ }^{9,10}$ Other studies have shown that EVAR accounts for a growing proportion of surgical procedures for AAA repair, while OSR is in relative decline. ${ }^{11}$ Similarly, some studies have shown wide variability in the criteria used to select the surgical technique. While selection of the specific surgical approach generally depends on the characteristics of the patient and the aneurysm itself, the unique characteristics of the treating hospital and the health care system may also play a role. This explains, in part, why treatment selection can be highly variable. ${ }^{12-14}$ Notwithstanding the aforementioned reports, relatively few studies have analyzed variability among hospitals in selection of the specific surgical approach for AAA. ${ }^{15-18}$ Such data would be valuable to identify differences among hospitals and countries with regard to the treatment options offered to patients with similar characteristics.

In this context, the research group "Atlas de Variabilidad de la Práctica Médica” (Atlas of Variations in Medical Practice; VPM in Spanish) carried out a study to assess the risk of death after surgery for AAA in-hospitals in the Spanish National Health Care System; this group also sought to identify differences in mortality rates among those hospitals. ${ }^{19,20}$ Using the data obtained by the VPM, we evaluated variation among Spanish hospitals with regard to the treatment of unruptured AAA. Specifically, we sought to identify current preferences among Spanish hospitals for OSR or EVAR and to determine changes in these preferences over the course of the study period (2002-2012).

\section{Methods}

\section{Study design, participants, and variables}

This was a retrospective, longitudinal study based on data from the year 2002 to 2012 in the database "Minimum Basic Dataset at Hospital Discharge" (in Spanish: "Conjunto Mínimo Básico de Datos al Alta Hospitalaria" [CMBD$\mathrm{AH}]$ ). This database - which was provided to us by the VPM - contains data on patients discharged from Spanish public hospitals. Specifically, we evaluated discharge data for patients $>18$ years old with a primary diagnosis of aortic aneurysm (AA) according to the ICD, Ninth Revision, Clinical Modification. The corresponding surgical procedure codes were also included and grouped as either OSR (codes 38.34, 38.44, 38.84, 39.25, 39.29, 39.23, 39.26, 39.51, 39.52, $39.57,39.56,39.58,38.46,38.66,38.86$, and 38.64.) or EVAR (codes 39.71, 39.73, 39.79, 39.90, 00.55, and 39.50). Inclusion criteria were as follows: 1) primary diagnosis of unruptured AAA, 2) elective admission, and 3) treated with
OSR or EVAR. The following patient and treatment-related data were registered: surgical hospital, gender, age at the time of surgery, and mortality at hospital discharge. The Charlson Comorbidity Index (CCI; using secondary diagnoses included in the CMBD) was also calculated and recorded.

Exclusion criteria were: patients diagnosed with thoracic or thoracoabdominal AA (codes: 441.1, 441.2, 441.4, 441.7 ) or aortic dissection (441.00, 441.01, 441.02, 441.03). We also excluded patients who underwent both EVAR and OSR during a single intervention, patients with ruptured AAA (codes: 441.3, 441.5, and 441.6), and patients with emergency admission.

\section{Statistical analyses}

A descriptive analysis of the main characteristics of the two surgical procedures was performed. Student's $t$-test was used to compare quantitative variables, while the chi-squared test or Fisher's exact test was used, as appropriate, to compare categorical variables. We used generalized linear mixed models (multivariate logistic regressions with hospital random effect) to examine interhospital variation. These models were run with and without adjustments to account for factors independently associated with surgical technique selection and in-hospital mortality. Three multivariate models that included temporal and hospital effects were developed. The first model included adjusted variables; the second model also considered a potential time trend (year of surgery); and the third model added surgical volume (ie, number of procedures) performed at each hospital. The results were adjusted for individual factors (age, gender, and comorbidities) to assess the influence of surgical volumes and compare improvement of the goodness of fit of the model (Hosmer-Lemeshow test).

Graphically, both the variation and the potential effect of surgical volumes were expressed using a funnel plot. ${ }^{21}$ Three statistical approaches were used to register variation: 1) intraclass correlation coefficient, using the latent variable approach to express the proportion of total variance explained by the treating center, 2) the median OR, and 3) the change in discriminatory power (receiver operating characteristic with area under the curve [AUC]) when using the center as the explanatory level.

The fixed effect of the individual and context variables were expressed as OR with 95\% CIs. Statistical significance was set at $P<0.05$. The statistical analysis was performed with the IBM-SPSS statistical software package, v25.0 (IBM Corp., Armonk, NY, USA), the Stata Statistical Software, release 15.2017 (Stata Corp.), and the R Core Team 2018 (R Foundation for Statistical Computing, Vienna, Austria). 


\section{Ethics approval and consent to participate}

Data used in the study were approved by the ethics committees of the participating institutions: "Atlas de Variaciones en la Práctica Médica" (Instituto Aragonés de Ciencias de la Salud) and University Hospital de la Santa Creu i Sant Pau (IIB Sant Pau). Informed consent was waived because we used anonymized retrospective data.

\section{Results}

A total of 30,372 patients underwent EVAR or OSR at 117 hospitals in Spain during the study period. Of these, 16,737 patients (55.1\%) (Figure 1) from 114 centers met the study inclusion criteria. A total of 6,809 patients (40.7\%) underwent EVAR and 9,928 (59.3\%) patients underwent OSR. Most patients were men $(16,185 ; 96.7 \%)$. The mean age was 71.4 years (SD 7.8). The mean CCI was 0.7 (SD 0.98). Variability among the participating centers in surgical volumes was high: the mean number of procedures per center during the study period was 328.2 (SD 159.25; range 1-682). Of the 16,737 patients, 601 died during the hospital stay, for an overall inhospital mortality rate of $3.6 \%$ (Table 1 ).

The total volume of surgical procedures at the participating hospitals increased from 1,132 procedures in 2002 to 1,859 in 2012, which represents a $64 \%$ increase overall and an annual increase of $5.8 \%$. The number of surgical procedures

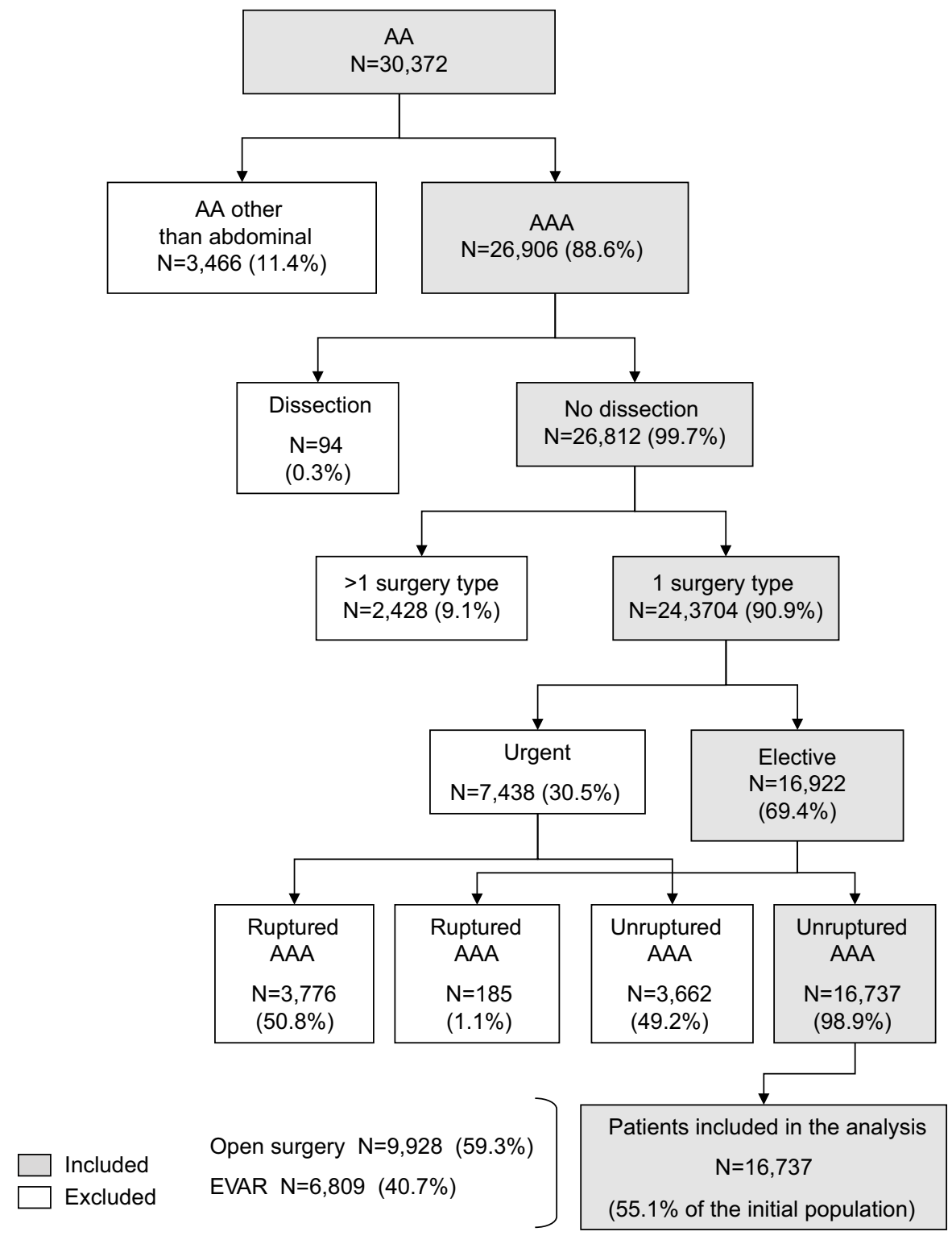

Figure I Selection process for study inclusion.

Note: Patients diagnosed with unruptured AAA, undergoing EVAR or OSR with an elective admission during the study period 2002-20I2.

Abbreviations: AA, aortic aneurysm; AAA, abdominal aortic aneurysm; EVAR, endovascular aneurysm repair; OSR, open surgical repair. 
per year increased progressively from 2002 to 2009, after which the volume stabilized.

Patients undergoing OSR were significantly younger than those treated with EVAR (OR 1.08, $P<0.001)$ and also had a significantly lower CCI (OR 1.16, $P<0.001)$. There was no significant association between gender and treatment selection (OR 1.14, $P=0.148$ ). However, there was a direct

Table I Characteristics of patients undergoing elective surgical repair for unruptured AAA in Spanish hospitals between 2002 and 2012

\begin{tabular}{|c|c|c|}
\hline & $\begin{array}{l}\text { Included pat } \\
(N=16,737)\end{array}$ & \\
\hline & $\mathbf{n}$ & $\%$ \\
\hline $\begin{array}{l}\text { Age (years) } \\
\text { Mean (SD) } \\
\text { Median (min-max) }\end{array}$ & $\begin{array}{l}71.4(7.8) \\
72(25-96)\end{array}$ & \\
\hline $\begin{array}{l}\text { Gender } \\
\text { Male } \\
\text { Female }\end{array}$ & $\begin{array}{l}16,185 \\
552\end{array}$ & $\begin{array}{l}96.7 \\
3.3\end{array}$ \\
\hline $\begin{array}{l}\text { Charlson Index } \\
\text { Mean (SD) } \\
\text { Median (min-max) }\end{array}$ & $\begin{array}{l}0.7(0.98) \\
0(0-10)\end{array}$ & \\
\hline $\begin{array}{l}\text { Type of intervention } \\
\text { EVAR } \\
\text { OSR }\end{array}$ & $\begin{array}{l}6,809 \\
9,928\end{array}$ & $\begin{array}{l}40.7 \\
59.3\end{array}$ \\
\hline $\begin{array}{l}\text { Surgical volumes for AAA repair per } \\
\text { hospital through the study period } \\
\text { Mean (SD) } \\
\text { Median (min-max) }\end{array}$ & $\begin{array}{l}328.2(159.25) \\
306(1-682)\end{array}$ & \\
\hline $\begin{array}{l}\text { In-hospital mortality rates } \\
\text { Yes } \\
\text { No }\end{array}$ & $\begin{array}{l}601 \\
16,136\end{array}$ & $\begin{array}{l}3.6 \\
96.4\end{array}$ \\
\hline
\end{tabular}

Abbreviations: AAA, abdominal aortic aneurysm; EVAR, endovascular aneurysm repair; OSR, open surgical repair. relation between surgical volumes and the use of EVAR. More specifically, the higher the volume, the greater the probability that the endovascular approach was used (OR $1.09, P<0.001$; Table 2). Nonetheless, there was substantial variability between surgical volume at any given hospital and the proportion of endovascular surgeries: in some cases, EVAR accounted for a relatively small percentage of procedures at high-volume hospitals, but a high percentage of AAA surgeries at hospitals with lower volumes (Figure 2A). Overall, the probability that any given procedure was EVAR increased by $\sim 20 \%$ annually (OR $1.20, P<0.001$; Figure $2 \mathrm{~B}$ ).

The overall in-hospital mortality rate in 2002 was $5.3 \%$, which declined to $3.2 \%$ in 2012 (OR 0.94, $P<0.001$ ), showing an annual decrease of $7 \%$ (Figure 3 ). This decrease in mortality rates was due primarily to the decrease in OSR-related mortality, with mortality rates falling from $6.3 \%$ in 2002 to $5.4 \%$ in 2012 (OR $0.98, P=0.235$ ); by contrast, the inhospital mortality increased slightly (but not significantly) in the EVAR group, from $0 \%$ in 2002 to $1.5 \%$ in 2012 (OR 0.97, $P=0.344)$.

At hospital discharge, the in-hospital mortality rate was significantly lower in the EVAR group vs OSR $(1.2 \%$ vs 5.2\%; OR 4.34, $P<0.001$ ). Two variables - age (OR 1.03, $P<0.001)$ and the CCI score (OR 1.26, $P<0.001)$ - were significantly associated with in-hospital mortality. There was no significant association between gender and mortality (OR $1.11, P=0.672)$. Higher annual surgical volumes were associated with lower mortality rates (OR $0.89, P<0.001$; Table 3 ).

Variability among the participating hospitals was high for the main study variables, which included the distribution of EVAR/OSR, in-hospital mortality rates, and surgical

Table 2 Characteristics of patients undergoing surgical repair for AAA: OSR vs EVAR

\begin{tabular}{|l|l|l|l|l|l|l|l|}
\hline \multicolumn{7}{|l|}{ Included patients (N=16,737) } \\
\hline
\end{tabular}

Abbreviations: AAA, abdominal aortic aneurysm; EVAR, endovascular aneurysm repair; OSR, open surgical repair. 

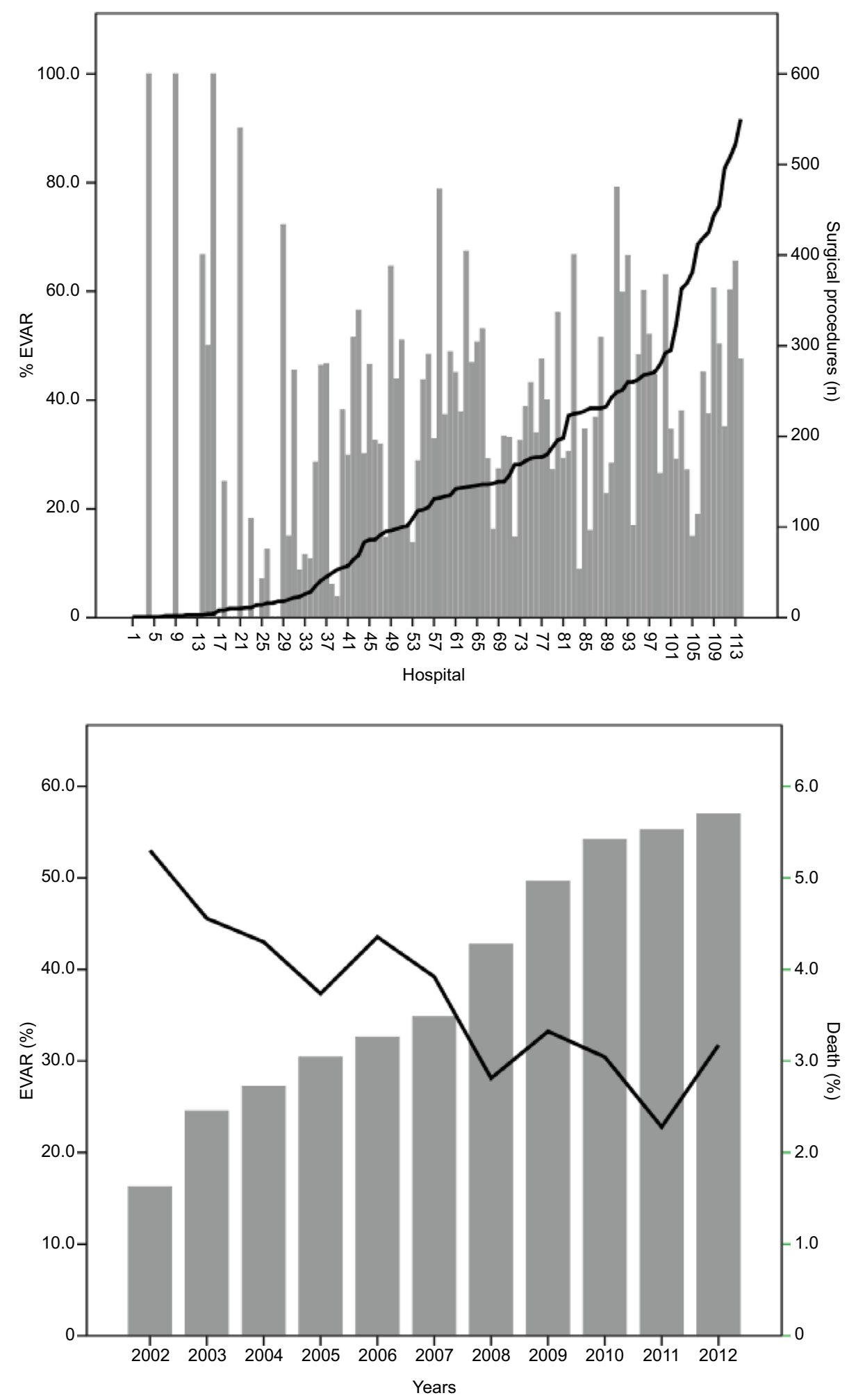

Figure 2 Volume of AAA surgeries performed in the hospitals participating in the study from 2002 to 2012, distribution of the two types of procedures and evolution of mortality.

Notes: (A) Number of surgical procedures performed at each participating hospital during the study period and the proportion of these interventions in each center. (B) EVAR as a proportion of surgical procedures to treat AAA from 2002 to 2012 at all participating hospitals. The mortality is also shown for the same period. Abbreviations: AAA, abdominal aortic aneurysm; EVAR, endovascular aneurysm repair. 


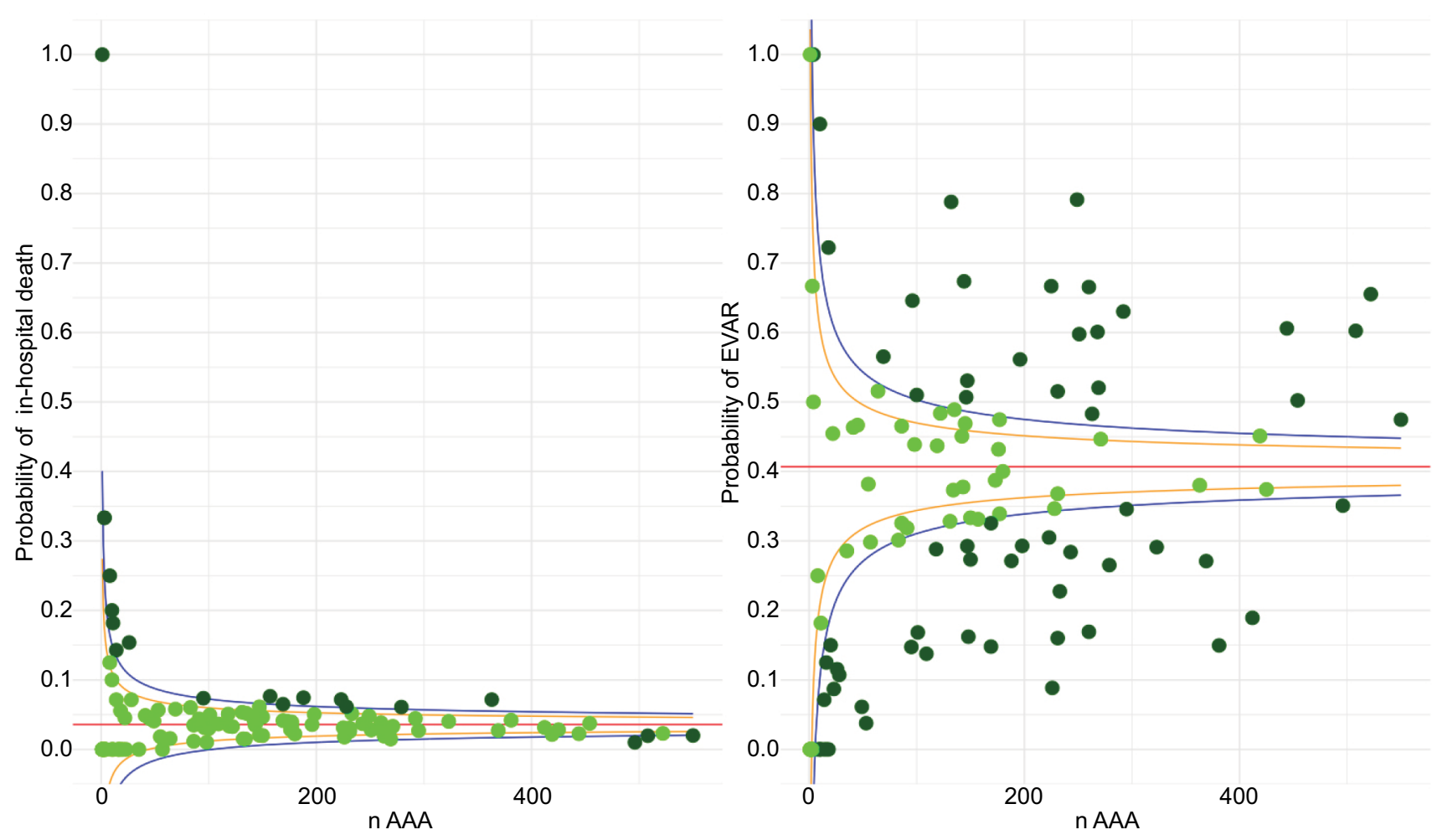

Figure 3 Funnel plot: probability of in-hospital mortality and surgery by EVAR, according to the volume of procedures performed in the hospitals participating in the study. Abbreviations: AAA, abdominal aortic aneurysm; EVAR, endovascular aneurysm repair.

Table 3 Mortality rates at discharge among patients undergoing surgical repair for AAA during the study period

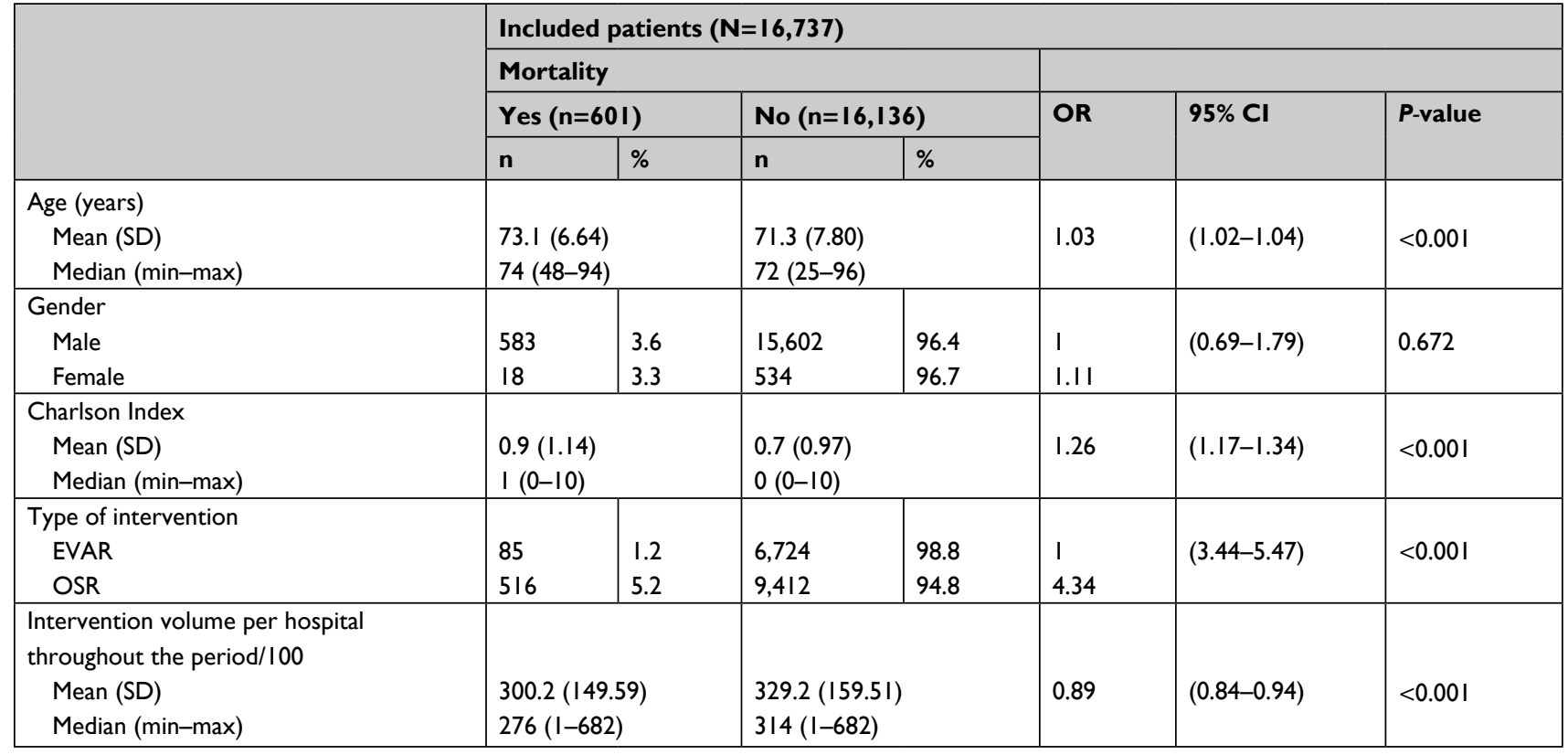

Abbreviations: AAA, abdominal aortic aneurysm; EVAR, indicates endovascular aneurysm repair; OSR, open surgical repair.

volumes. Variability in preferences for EVAR or OSR was particularly high, with a large percentage of values falling outside the expected range. Mortality, however, did not show such a high variability (Figure 3).
The multivariate analysis, which was based on the three different models, revealed a significant and increasing trend over time toward the greater use of EVAR vs OSR (OR 1.25). Nevertheless, despite this clear trend toward the use of this 
minimally invasive technique, we did not observe any corresponding decrease in in-hospital deaths among patients treated with EVAR compared to those treated with OSR (OR 0.98). Higher-volume hospitals were more likely to perform EVAR (OR 1.38), and mortality rates were lower in the highvolume hospitals (OR 0.87; Table 4).

The hospital effect - the influence that the variable "hospital" itself had on the choice of surgical technique - was highly relevant, accounting for almost $20 \%$ of the variance in this variable. However, this same effect accounted for only slightly over $2 \%$ of the differences in in-hospital mortality. In other words, the influence of hospital characteristics is much greater on determining the type of surgical procedure that is performed than on patients' mortality. The median OR for the hospital effect on treatment selection was 2.4, with an OR of 1.3 for immediate mortality. Overall, the capacity of the model to discriminate for mortality was good (AUC $=0.74)$; by contrast, the discriminatory capacity for treatment selection was poor $(\mathrm{AUC}=0.50)$, as shown in Table 4 .

\section{Discussion}

The present study was carried out to analyze variation among Spanish hospitals in the choice of treatment - OSR or EVAR - for elective AAA surgery and to assess changes over time in these preferences. Our findings showed that both the surgical approach and surgical volumes vary widely across Spanish hospitals. During the study period, the overall number of elective surgical procedures for AAA increased by $64 \%$, an annual increase of 5.8\%, with EVAR accounting for a growing proportion of those surgical procedures. Overall in-hospital mortality rates decreased during the study period, primarily due to a decrease in OSR-related mortality, suggesting improved safety. Not surprisingly, higher-volume centers had better results in terms of lower in-hospital mortality rates, which was independent of the predominant surgical technique at the institution. These results are consistent with previous reports, ${ }^{9}$ thus confirming the growing number of surgical indications for patients with AAA and the increasing preference for EVAR vs OSR. ${ }^{11}$

During the 11-year study period, the volume of elective AAA procedures conducted in Spanish public hospitals (not including emergency procedures and cases with ruptured AAA) increased by $64 \%$. While EVAR accounted for $38 \%$ of all AAA procedures performed during the study period, the use of EVAR increased by $40 \%$ from 2002 to 2012, was $16 \%$ at the beginning of the period, and eventually accounted for $>56 \%$ of all surgical procedures for AAA in the year 2012 . Factors such as older age, more comorbidities, and elective admission all increased the likelihood that a patient would undergo EVAR rather than OSR. In general, EVAR was more common than OSR in higher-volume hospitals, although this was highly variable, as some high-volume centers presented a low EVAR to OSR ratio. This is an important finding, as it indicates high inter-center variability in the selection of the surgical procedure.

Surgical volumes varied widely among the participating hospitals, ranging from as low as only a single AAA surgery at one hospital to as many as 682 surgeries at the most active

Table 4 Multilevel logistic regression analysis of surgery selection and in-hospital mortality in public hospitals in Spain, 2002-2012

\begin{tabular}{|c|c|c|c|c|c|c|}
\hline & \multicolumn{3}{|c|}{ Selection of EVAR } & \multicolumn{3}{|c|}{ In-hospital mortality } \\
\hline & Model I & Model 2 & Model 3 & Model I & Model 2 & Model 3 \\
\hline \multicolumn{7}{|c|}{ Specific individual average effects } \\
\hline Age (years) & $1.90(1.82-1.97)$ & $1.93(1.85-2.01)$ & $1.89(1.82-1.97)$ & $1.58(1.43-1.74)$ & $1.58(1.43-1.74)$ & $1.58(1.43-1.74)$ \\
\hline Female vs male & $1.02(0.84-1.23)$ & $0.94(0.77-1.15)$ & $1.02(0.84-1.24)$ & $0.89(0.55-1.44)$ & $0.90(0.55-1.46)$ & $0.89(0.55-1.44)$ \\
\hline Charlson Index & $1.14(1.10-1.18)$ & $1.12(1.08-1.16)$ & $1.13(1.10-1.18)$ & $1.27(1.19-1.37)$ & $1.28(1.20-1.37)$ & $1.27(1.19-1.37)$ \\
\hline EVAR vs OSR & -------- & - ------ & ------- & $0.17(0.13-0.22)$ & $0.18(0.14-0.22)$ & $0.17(0.14-0.22)$ \\
\hline Year & & $1.25(1.23-1.26)$ & & & $0.98(0.95-1.01)$ & \\
\hline \multicolumn{7}{|c|}{ Specific contextual average effects } \\
\hline Volume of intervention & & & $1.38(1.14-1.67)$ & & & $0.87(0.79-0.97)$ \\
\hline \multicolumn{7}{|c|}{ General contextual effects } \\
\hline ICC (\%) & 21.04 & 22.27 & 19.89 & 2.86 & 2.86 & 2.31 \\
\hline Median OR & 2.44 & 2.56 & 2.37 & 1.35 & 1.35 & 1.30 \\
\hline AUC & $0.5 \mathrm{I}(0.49-0.53)$ & $0.48(0.46-0.50)$ & $0.5 \mathrm{I}(0.49-0.53)$ & $0.74(0.72-0.76)$ & $0.74(0.72-0.76)$ & $0.74(0.72-0.76)$ \\
\hline \multicolumn{7}{|l|}{ Goodness of fit } \\
\hline $\mathrm{DIC}$ & $19,649.96$ & $18,36 \mid .55^{*}$ & $19,640.54$ & $4,827.75$ & $4,827.94$ & $4,824.34^{*}$ \\
\hline
\end{tabular}

Notes: Values are given as OR with $95 \% \mathrm{Cl}$, unless stated otherwise. The intercept is not shown in the table. *Significant change relative to model I (likelihood ratio: $P<0.05$ ). Abbreviations: AUC, area under the receiver operating characteristic curve; DIC, Bayesian deviance information criterion; EVAR, endovascular aneurysm repair; ICC, intraclass correlation coefficient; OSR, open surgical repair. 
center. Similarly, preferences for EVAR vs OSR were also highly variable. EVAR accounted for anywhere from $0 \%$ to $100 \%$ of all elective AAA surgeries, depending on the hospital. These heterogeneous findings are consistent with the results of a large international study ${ }^{12}$ that found that the therapeutic management of AAA varies widely from country to country and even across regions within the same country. Consistent with our findings, that study showed wide variability between hospitals in terms of both surgical volumes and in the proportion of patients undergoing EVAR. This wide variability is somewhat surprising, given the similarity of the clinical practice recommendations published by most professional societies. ${ }^{12}$

Inter-center variability in the surgical approach (ie, EVAR vs OSR) is probably due to differences in selection criteria. It seems likely that the choice of technique could affect outcomes, primarily complication and mortality rates. The selection of one technique or another typically depends on patient characteristics and on the anatomic characteristics of the aneurysm itself; however, treatment selection also depends on the treating center and on the surgeons' expertise, which is why variability can be wide. ${ }^{22}$

The findings of the present study are consistent with previous reports that have found EVAR to be associated with lower in-hospital mortality rates than open surgery. Several factors are associated with an increased risk of mortality, including age, female gender, presence of comorbidities, and a low surgical volume at the treating center. The ATLAS VPM group conducted a study that was similar to the present study, but with fewer hospitals and patients and with a shorter follow-up. ${ }^{20}$ Not surprisingly - given that both studies were based on a similar data set - the demographic characteristics and distribution of the two types of surgery in that study were similar to our data. However, whereas we found that hospital volume independently (ie, regardless of the mix of surgical techniques) affected in-hospital mortality (adjusted by age, gender, and comorbidity), the ATLAS VPM study found that lower mortality rates were mainly attributable to the use of EVAR, and that this decrease was independent of age, comorbidities, or annual surgical volume at the treating center.

We found that in-hospital mortality decreased over the 11-year study period, mainly attributable to the decrease in mortality associated with OSR. These results probably reflect improvements in the OSR technique or better patient selection. In terms of EVAR, we found a slight increase in mortality, perhaps due to the overuse of this technique related to the rapid and progressive expansion of the indications for this procedure. ${ }^{1}$ Surprisingly, both of these findings contradict the results of an international study conducted by Budtz-Lilly et al. ${ }^{23}$ That study analyzed data from vascular surgery records in eleven countries, finding that OSR-related mortality had increased during the study period, whereas EVAR-related mortality rates had decreased. Although the reason for the differences between the two studies is not clear, perhaps these contrasting findings could be explained (at least partially) by differences in the sources of information used (a large database from the Ministry of Health vs medical records) or the criteria used to select the surgical technique.

To better understand how temporal factors or surgical volumes influence treatment-related outcomes, we performed a multilevel analysis adjusted for patient characteristics. This analysis showed that the volume of surgical procedures at the treating center was independently associated with a preference for EVAR. In other words, the greater the volume, the higher the probability of selecting EVAR. Higher surgical volumes were also associated with lower in-hospital mortality rates: centers that performed more surgical procedures (EVAR or OSR) had lower in-hospital mortality rates. This association between a higher volume of AAA procedures and better treatment outcomes is consistent with previous reports. ${ }^{24,25}$ Both our findings and those of other studies show that immediate mortality rates are lower in higher volume hospitals, regardless of the specific surgical procedure. Some studies have even demonstrated a clear relationship between the number of surgeries performed by the surgeon and mortality rates, regardless of the specific number of surgeries performed annually at the center. ${ }^{12,22,24,25}$

It has long been known that centers with higher volumes achieve better results overall, especially in complex, highrisk, and uncommon surgeries (particularly in cardiology and oncology). ${ }^{26}$ Mortality rates are lower and overall outcomes are better in patients treated in-hospital with a large number of patients with the same condition compared to those treated in less-experienced centers. ${ }^{27,28}$ In the context of surgical treatment of AAA, our findings - and those of other studies support the implementation of policies to concentrate elective AAA surgical procedures at reference centers, which would ensure a higher volume of cases and better outcomes. ${ }^{24,29}$

\section{Study strengths and limitations}

An important limitation of the present study is that we were unable to determine whether the selected procedure was appropriate for the patient based on clinical and anatomic criteria. This determination was not possible because the data set did not include the necessary information to assess this factor. Similarly, because the data set contained only data up to hospital discharge, we were unable to perform any additional 
follow-up analyses. In this regard, it would be valuable to perform a study in the future to determine the suitability of the specific surgical technique used to treat patients with AAA. An important strength of this study is that the findings are based on consistent data from discharge data obtained from nearly all Spanish hospitals for a long period of time (11 years). Moreover, the study included $>16,000$ patients.

\section{Conclusion}

The present study shows that variability among Spanish hospitals in terms of the surgical management of AAA repair is high. Surgical volumes and treatment selection are both highly variable among centers. The number of surgical procedures to repair AAA increased substantially from 2002 to 2012, with EVAR comprising a growing proportion of all procedures. Overall in-hospital mortality decreased significantly during this time period, mainly among patients treated with OSR. High-volume centers had better outcomes - both for EVAR and OSR - with lower immediate mortality rates.

Ideally, controlled clinical trials should be conducted to compare treatment outcomes achieved with new endovascular devices vs those obtained with OSR. Data from such trials would be valuable to assess the suitability of concentrating elective AAA repair in more experienced centers to potentially achieve better outcomes.

\section{Data sharing statement}

Please contact the corresponding author.

\section{Acknowledgments}

The authors would like to thank Andrea Cervera Alepuz and Bradley Londres for their help in editing the final version of the manuscript. This work was supported by a grant from the Instituto de Salud Carlos III (PI12/02530). The authors would also like to acknowledge the AAA Spanish Study Group.

\section{Disclosure}

The authors report no conflicts of interest in this work.

\section{References}

1. Healey CT, Neilson M, Clark D, Schanzer A, Robinson W; Vascular Study Group of New England None. Predicting mortality of ruptured abdominal aortic aneurysms in the era of endovascular repair. Ann Vasc Surg. 2017;38:59-63.

2. Wennberg J, Gittelsohn A. Small area variations in health care delivery: a population-based health information system can guide planning and regulatory decision-making. Science. 1973;182(4117):1102-1108.

3. Moll FL, Powell JT, Fraedrich G, et al. Management of abdominal aortic aneurysms clinical practice guidelines of the European Society for vascular surgery. Eur J Vasc Endovasc Surg. 2011;41(Suppl 1): S1-S58.
4. Multicentre Aneurysm Screening Study Group. Multicentre Aneurysm Screening study (MASS): cost effectiveness analysis of screening for abdominal aortic aneurysms based on four year results from randomised controlled trial. BMJ. 2002;325(7373):1135-1138.

5. Cosford PA, Leng GC. Screening for abdominal aortic aneurysm. Cochrane Database Syst Rev. 2007(2):CD002945.

6. Svensjö S, Mani K, Björck M, Lundkvist J, Wanhainen A. Screening for abdominal aortic aneurysm in 65-year-old men remains cost-effective with contemporary epidemiology and management. Eur J Vasc Endovasc Surg. 2014;47(4):357-365.

7. Franks SC, Sutton AJ, Bown MJ, Sayers RD. Systematic review and meta-analysis of 12 years of endovascular abdominal aortic aneurysm repair. Eur J Vasc Endovasc Surg. 2007;33(2):154-171.

8. Blankensteijn JD, de Jong SECA, Prinssen M, et al; Dutch Randomized Endovascular Aneurysm Management (DREAM) Trial Group. Two-year outcomes after conventional or endovascular repair of abdominal aortic aneurysms. N Engl J Med. 2005;352(23):2398-2405.

9. Drury D, Michaels JA, Jones L, Ayiku L. Systematic review of recent evidence for the safety and efficacy of elective endovascular repair in the management of infrarenal abdominal aortic aneurysm. Br J Surg. 2005;92(8):937-946.

10. Wendt K, Kristiansen R, Krohg-Sørensen K, Gregersen FA, Fosse E. Trends in abdominal aortic and iliac aneurysm repairs in Norway from 2001 to 2013. Eur J Vasc Endovasc Surg. 2016;51(2):194-201.

11. Levin DC, Rao VM, Parker L, Frangos AJ, Sunshine JH. Endovascular repair vs open surgical repair of abdominal aortic aneurysms: comparative utilization trends from 2001 to 2006. JAm Coll Radiol. 2009;6(7):506-509.

12. Schanzer A, Steppacher R, Eslami M, Arous E, Messina L, Belkin M. Vascular surgery training trends from 2001-2007: a substantial increase in total procedure volume is driven by escalating endovascular procedure volume and stable open procedure volume. J Vasc Surg. 2009;49(5):1339-1344.

13. Beck AW, Sedrakyan A, Mao J, et al. Variations in abdominal aortic aneurysm care: a report from the International consortium of vascular registries. Circulation. 2016;134(24):1948-1958.

14. Fitridge RA, Boult M, Mackillop C, et al. International trends in patient selection for elective endovascular aneurysm repair: sicker patients with safer anatomy leading to improved 1-year survival. Ann Vasc Surg. 2015;29(2):197-205.

15. Schermerhorn ML, Buck DB, O'Malley AJ, et al. Long-term outcomes of abdominal aortic aneurysm in the Medicare population. $N$ Engl $J$ Med. 2015;373(4):328-338.

16. Zettervall SL, Buck DB, Soden PA, et al; Society for Vascular Surgery Vascular Quality Initiative. Regional variation exists in patient selection and treatment of abdominal aortic aneurysms. J Vasc Surg. 2016;64(4):921-927.

17. Zettervall SL, Soden PA, Buck DB, et al; Dutch Randomized Endovascular Aneurysm Management (DREAM) Trial Group. Significant regional variation exists in morbidity and mortality after repair of abdominal aortic aneurysm. J Vasc Surg. 2017;65(5):1305-1312.

18. Tambyraja AL, Rodriguez-Lopez JA, Ramaiah V, Diethrich EB, Chalmers RT. Institutional differences in endovascular aneurysm repair and aneurysm morphology. World J Surg. 2012;36(11): $2738-2741$.

19. VPM Atlas [homepage on the Internet]. Project VPM Atlas Variations in Medical Practice in the National Health System. Available from: http://www.atlasvpm.org/home. Accessed October 18, 2018

20. Variabilidad en el Riesgo de Morir tras sufrir intervención sobre Aneurisma de Aorta Abdominal Integro en hospitales de agudos del Sistema Nacional de Salud. Documento de trabajo 01-2009. Grupo ATLAS VPM. Plan de Calidad para el Sistema Nacional de Salud. Spain.

21. Spiegelhalter DJ. Funnel plots for comparing institutional performance. Stat Med. 2005;24(8):1185-1202.

22. McOhee JT, Robinson WP, Eslami MH, Arous EJ, Messina LM, Schanzer A. Surgeon case volume, not institution case volume, is the primary determinant of in-hospital mortality after elective open abdominal aortic aneurysm repair. J Vasc Surg. 2011;53(3):591-599. 
23. Budtz-Lilly J, Venermo M, Debus S, et al. Editor's choice - assessment of international outcomes of intact abdominal aortic aneurysm repair over 9 years. Eur J Vasc Endovasc Surg. 2017;54(1):13-20.

24. Dimick JB, Upchurch GR. Endovascular technology, hospital volume, and mortality with abdominal aortic aneurysm surgery. JVasc Surg. 2008;47(6): 1150-1154.

25. Holt PJE, Poloniecki JD, Gerrard D, Loftus IM, Thompson MM. Meta-analysis and systematic review of the relationship between volume and outcome in abdominal aortic aneurysm surgery. Br J Surg. 2007;94(4):395-403.

26. Pieper D, Mathes T, Neugebauer E, Eikermann M. State of evidence on the relationship between high-volume hospitals and outcomes in surgery: a systematic review of systematic reviews. J Am Coll Surg. 2013;216(5):e18:1015-1025.
27. Gordon TA, Burleyson GP, Tielsch JM, Cameron JL. The effects of regionalization on cost and outcome for one general high-risk surgical procedure. Ann Surg. 1995;221(1):43-49.

28. Birkmeyer JD, Siewers AE, Finlayson EVA, et al. Hospital volume and surgical mortality in the United States. $N$ Engl J Med. 2002;346(15): 1128-1137.

29. Eckstein HH, Bruckner T, Heider P, et al. The relationship between volume and outcome following elective open repair of abdominal aortic aneurysms (AAA) in 131 German hospitals. Eur JVasc Endovasc Surg. 2007;34(3):260-266. 


\section{Supplementary material}

\section{AAA Spanish Study Group members}

Hospital de la Santa Creu i Sant Pau, IIB Sant Pau, Barcelona, Spain. CIBER of Epidemiology and Public Health (CIBERESP), Spain. Iberoamerican Cochrane Center, Barcelona, Spain: Xavier Bonfill, M Jesús Quintana. Hospital de la Santa Creu i Sant Pau, IIB Sant Pau, Barcelona, Spain. CIBER of Cardiovascular Diseases (CIBERCV), Spain: José R Escudero. Vall d'Hebron University Hospital, Barcelona, Spain. Vall d'Hebron Research Institute (VHIR), Barcelona, Spain: Sergi Bellmunt. Hospital Ramón y Cajal, Madrid, Spain. Health Research Institute (IRYCIS), Spain. CIBER of Epidemiology and Public Health (CIBERESP), Spain: Nieves Plana, Alfonso Muriel, Borja Castejón. Hospital La Fe, Valencia, Spain: Manuel Miralles, Laura Gálvez Núñez. University of Valencia, Valencia, Spain. CIBER of Epidemiology and Public Health (CIBERESP), Spain: Maria M Morales-Suárez-Varela. Hospital 12 de Octubre, Madrid, Spain: Inés Fernández de Valderrama, Ana Isabel Rodríguez. Hospital 12 de Octubre, Madrid, Spain. CIBER of Epidemiology and Public Health (CIBERESP), Spain: Agustín Gómez de la Cámara. Hospital Cruces, Barakaldo, Spain. Biocruces Health Research Institute, Barakaldo, Spain. CIBER of Epidemiology and Public Health (CIBERESP), Spain: José Ignacio Pijoan; Eduardo Pérez.
Vascular Health and Risk Management

\section{Publish your work in this journal}

Vascular Health and Risk Management is an international, peerreviewed journal of therapeutics and risk management, focusing on concise rapid reporting of clinical studies on the processes involved in the maintenance of vascular health; the monitoring, prevention and treatment of vascular disease and its sequelae; and the involvement of

\section{Dovepress}

metabolic disorders, particularly diabetes. This journal is indexed on PubMed Central and MedLine. The manuscript management system is completely online and includes a very quick and fair peer-review system, which is all easy to use. Visit http://www.dovepress.com/ testimonials.php to read real quotes from published authors.

Submit your manuscript here: https://www.dovepress.com/vascular-health-and-risk-management-journal 